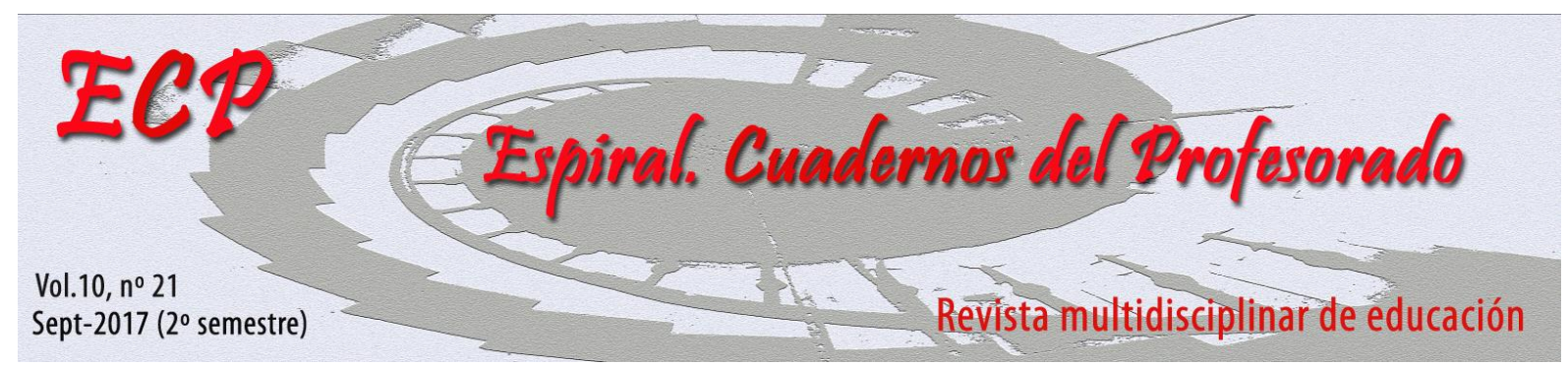

\title{
Comparación social de la apariencia en contextos de ejercicio físico como variable predictora de los trastornos de la conducta alimentaria en adolescentes de ambos sexos
}

\section{Social appearance comparison in exercise contexts as a predictor of eating disorder symptoms in male and female adolescents}

\author{
Manuel Alcaraz-Ibáñez
}

Universidad de Almería

\begin{abstract}
RESUMEN: El objetivo de este estudio fue investigar la influencia de la comparación de la apariencia realizada en contextos de práctica de ejercicio físico sobre el riesgo de presencia de trastornos de la conducta alimentaria en población adolescente, considerando a su vez el posible papel moderador de diversas variables previamente asociadas con dicho desorden. Un total de 236 escolares $\left(117\right.$ chicos y 119 chicas; $M_{\text {edad }}=13.78, D T=1.15$ ) tomaron parte en el estudio. Aplicando una técnica de bootstrapping, se realizaron diversos análisis de regresión y moderación empleando el macro PROCESS para SPSS. Los resultados mostraron que, tras controlar los efectos del sexo, la edad, el índice de masa corporal, y la insatisfacción corporal, la comparación de la apariencia realizada al practicar ejercicio físico se asociaba de forma positiva y estadísticamente significativa con la presencia de síntomas propios de los trastornos de la conducta alimentaria tanto en chicos como en chicas. Los resultados del análisis de moderación mostraron que la percepción de insatisfacción corporal intensificaba dicha relación, contribuyendo de forma estadísticamente significativa a explicar un $4 \%$ adicional de varianza en el modelo. Las evidencias obtenidas sugieren que la comparación de la apariencia realizada en contextos de práctica de ejercicio físico es un elemento que debería ser considerado a la hora de diseñar programas destinados a la prevención de la aparición de trastornos de la conducta alimentaria en población adolescente de ambos sexos. A la luz de estos resultados, tanto profesores de Educación Física como monitores y entrenadores deportivos que trabajen con población adolescente deberían tratar de evitar en su práctica profesional situaciones que pudiesen promover o facilitar la comparación de la apariencia física por parte de sus pupilos.
\end{abstract}

Palabras clave: trastornos de la conducta alimentaria, adolescentes, ejercicio físico, comparación social, educación física, imagen corporal.

\begin{abstract}
This study aims to investigate the influence of appearance social comparison made in exercise contexts on the risk of developing an eating disorder in adolescent population taking as well into consideration the potential moderating role of several variables previously associated with this disorder. A total of 236 students (117 boys and 119 girls; $M_{\text {age }}=13.78, D T=1.15$ ) participated in this study. Bootstrapping regression and moderation analyses using the PROCESS macro for SPSS were performed. Results showed that, after controlling for the effects of sex, age, body mass index, and body dissatisfaction, social appearance comparison was positively and statistically significantly associated with the presence of eating disorder symptoms in both girls and boys. Moreover, the results of the moderation analysis shown that body dissatisfaction strengthened this relationship, thus significantly explaining and additional $4 \%$ of the variance in the model. Provided evidence suggests that social appearance comparison made in exercise contexts should be targeted by health promotion programs aimed to prevent disordered eating for both boys and girls. In light of these results, both physical education teachers and sports instructors working with adolescents should try to avoid in their professional practice situations that might promote or facilitate physical appearance comparison of their pupils.
\end{abstract}

Key words: eating disorders, adolescents, exercise, social comparison, physical education, body image. 
Alcaraz-Ibáñez, M. (2017). Comparación social de la apariencia en contextos de ejercicio físico como variable predictora de los trastornos de la conducta alimentaria en adolescentes de ambos sexos. Espiral. Cuadernos del Profesorado, 10(21), 80-89.

Fecha de recepción: 12/07/2017

Fecha de aceptación: 17/09/2017
Correspondencia: manuelalcaraz74@gmail.com

(M. Alcaraz-Ibáñez)

\section{Introducción}

Durante la adolescencia, el ser humano experimenta una serie de profundas transformaciones que, en algunos casos, pueden dar lugar a la aparición de diversos trastornos de carácter psico-afectivo y conductual (DiClemente, Hansen, \& Ponton, 1996). De entre este tipo de trastornos, el grupo de los relacionados con la conducta alimentaria (TCA) se muestra como uno de los más prevalentes (Klump, 2013). En este sentido, estudios que consideraron muestras de adolescentes europeos de entre 12 y 18 años sugieren que entre el 8-18\% de los chicos y el 22-31\% de las chicas podrían encontrarse en riesgo de sufrir un TCA (Ferreiro, Seoane, \& Senra, 2012; Herpertz-Dahlmann, Dempfle, Konrad, Klasen, \& Ravens-Sieberer, 2015; Veses et al., 2014). Estas cifras resultan especialmente preocupantes, en concreto, atendiendo a las evidencias que sugieren que experimentar un TCA durante la adolescencia podría aumentar el riesgo de sufrir sobrepeso, obesidad y depresión durante las primeras etapas de la edad adulta (Herpertz-Dahlmann et al., 2015). En función de estos antecedentes, la necesidad de profundizar en la prevención y la detección temprana de los TCA parece justificada.

La literatura científica sugiere que los factores de riesgo o desencadenantes de la aparición de los TCA pueden ser de distinta naturaleza. En esta línea, el modelo socio-ecológico de influencia (Wang, Peterson, McCormick, \& Austin, 2013) señala dos principales grupos de factores. Así, en un primer grupo se incluirían el conjunto de influencias de carácter sociocultural presentes en los distintos contextos vitales (e.g., escolar o familiar); este sería el caso de la existencia de determinados modelos o ideales de cuerpo, o la exposición tanto a los medios de comunicación como a las influencias de carácter inter-personal ejercidas, por ejemplo, por familiares, amistades y profesorado. A su vez, en un segundo grupo se incluirían el conjunto de diferencias individuales de carácter demográfico (e.g., sexo o edad) o psicosocial inherentes a cada persona. En este sentido, uno de los factores de riesgo psicosocial que según la investigación previa podría contribuir a la aparición de los TCA es la tendencia a la comparación social (Fitzsimmons-Craft et al., 2014; Fitzsimmons-Craft, Bardone-Cone, \& Harney, 2012; Schaefer \& Thompson, 2014).

De acuerdo a los postulados de la teoría de comparación social (Festinger, 1954), el ser humano tiende de manera innata a autoevaluarse en función de la información obtenida al compararse con sus semejantes. En esta línea, los resultados de anteriores investigaciones han asociado la comparación social de la propia apariencia con el riesgo de presencia de TCA. Por ejemplo, Fitzsimmons-Craft et al. $(2014,2012)$ considerando muestras de mujeres de entre 17 y 27 años, así como Schaefer y Thompson (2014) considerando una muestra de mujeres de entre 17 y 58 años, observaron la existencia de una relación positiva entre la frecuencia en la comparación de la apariencia y el riesgo de aparición de TCA. A su vez, dichos trabajos mostraron que, una vez controlados los efectos del índice de masa corporal (IMC), la frecuencia en la comparación de la apariencia contribuía a explicar la sintomatología propia de los TCA. De igual forma, Halliwell y Harvey (2006) informaron de la existencia de una relación positiva entre la frecuencia en la comparación social de la apariencia y el riesgo de presencia de TCA en una muestra de adolescentes de ambos sexos de entre 11 y 16 años de edad.

Pese a que las evidencias anteriormente expuestas sugieren que la tendencia a la comparación de la apariencia podría contribuir al riesgo de aparición de TCA, cabe destacar que éstas fueron obtenidas, en la mayoría de los casos, considerando muestras formadas por mujeres adultas o en las últimas fases de la adolescencia (Fitzsimmons-Craft et al., 2014, 2012; Schaefer \& Thompson, 2014) en lugar de en población adolescente (Halliwell \& Harvey, 2006). Esta limitación resulta relevante por cuanto la adolescencia se muestra como un periodo del ciclo vital especialmente proclive a la 
aparición de los TCA (Klump, 2013). Además, pese a que los TCA se muestran menos prevalentes en chicos que en chicas, la investigación señala que este tipo de desórdenes también afectan a un número importante de chicos (Ferreiro et al., 2012; Veses et al., 2014). De igual forma, cabe destacar que el contenido de las herramientas empleadas en los trabajos que previamente consideraron la relación entre la comparación de la apariencia y la presencia de TCA aludían tanto a la tendencia a la comparación realizada de manera genérica (Fitzsimmons-Craft et al., 2014, 2012; Halliwell \& Harvey, 2006) como a diferentes contextos y situaciones en los cuales dicha comparación podría producirse (Schaefer \& Thompson, 2014). No obstante, algunas de estas situaciones podrían no llegar a presentarse en adolescentes de menor edad (e.g., "Cuando estoy comiendo en un restaurante, comparo mi nivel de grasa corporal con el de los demás"). A su vez, las posibilidades de intervenir al objeto de controlar la tendencia a la comparación de la apariencia en algunas de las situaciones y contextos planteados (e.g., "Cuando estoy en una fiesta comparo la forma de mi cuerpo con la de los demás") podrían ser muy limitadas.

Uno de los contextos en los cuales la comparación de la apariencia ha sido asociada con el riesgo de desarrollar un TCA es el de la práctica de ejercicio físico. No obstante, las evidencias obtenidas en dicho contexto no han estado referidas de manera específica a la comparación de la apariencia; por contra, éstas aludían a características propias de la práctica de ejercicio físico como, por ejemplo, su duración o intensidad (Fitzsimmons-Craft et al., 2014, 2012). Sin embargo, el estudio de los hábitos de comparación de la apariencia en el contexto de la práctica de ejercicio físico podría resultar de especial interés en población adolescente, ya que aunque no todos los individuos pertenecientes a este grupo poblacional practiquen necesariamente ejercicio físico en su tiempo libre, éstos sí que participan en las clases de Educación Física durante la etapa de Educación Secundaria. De esta forma, la gran mayoría de los adolescentes de entre 12-17 años podrían encontrarse potencialmente expuestos a este tipo de comparación. Pese a esto, la posible influencia que la comparación social de la propia apariencia producida de manera específica en contextos de práctica de ejercicio físico sobre el riesgo de desarrollar un TCA no ha sido aún investigada. Obtener evidencias que apoyasen dicha relación permitiría identificar un factor de riesgo que podría ser considerado en la práctica docente en una etapa del ciclo vital tan sensible para la adquisición de futuros hábitos como es la adolescencia. De hecho, la imagen corporal constituye un elemento transversal para las diferentes materias del currículum contempladas en la vigente y actual Ley Orgánica para la Mejora de la Calidad Educativa (LOMCE) en España.

El principal objetivo del presente trabajo fue observar la posible influencia de la frecuencia en la comparación social de la propia apariencia, realizada por parte de los adolescentes en el contexto de la práctica de ejercicio físico, sobre el riesgo de presencia de TCA. Atendiendo a los resultados de anteriores estudios que consideraron muestras formadas exclusivamente por mujeres (FitzsimmonsCraft et al., 2014, 2012; Schaefer \& Thompson, 2014), se hipotetizó que la comparación de la propia apariencia y, en concreto, la ocurrida en el contexto de la práctica de ejercicio físico, contribuiría a explicar el riesgo de aparición de TCA tanto en chicas como en chicos. A su vez, se espera que dicha relación se produzca una vez controlados los efectos que sobre el riesgo de presencia de TCA podrían ejercer variables como sexo (Ferreiro et al., 2012; Veses et al., 2014), IMC (Ferreiro et al., 2012; Fitzsimmons-Craft et al., 2014, 2012; Schaefer \& Thompson, 2014), edad (Ferreiro et al., 2012), o insatisfacción corporal (Ferreiro et al., 2012; Rohde, Stice, \& Marti, 2015). La investigación previa sugiere que los factores que contribuyen a la aparición de los TCA podrían no resultar universalmente válidos, de forma que éstos podrían variar en función de variables como el sexo (Ferreiro et al., 2012; Mayer-Brown, Lawless, Fedele, Dumont-Driscoll, \& Janicke, 2016) o la edad (Rohde et al., 2015). De igual forma, existen evidencias que sugieren que el estudio de la interacción entre los distintos tipos de factores que intervienen en la aparición de los TCA podría contribuir a una mayor comprensión de los mecanismos que desencadenan este tipo de trastornos en población adolescente (Mayer-Brown et al., 2016). Por consiguiente, se consideró el posible papel moderador que variables como sexo, edad, IMC, o insatisfacción corporal, podrían ejercer en la relación entre la comparación social de la apariencia en contextos de ejercicio físico y el riesgo de padecer un TCA. 
Como objetivo secundario, se estudió la posible existencia de diferencias entre chicas y chicos, tanto en los niveles de frecuencia en la comparación social de la apariencia en contextos de práctica de ejercicio físico, como en el riesgo de presencia de TCA. Se hipotetizó que, al igual que en anteriores estudios donde la frecuencia en la comparación de la apariencia fue considerada de forma general (Halliwell \& Harvey, 2006), las chicas mostrarían mayores niveles en dicha variable; en el caso del presente estudio, considerada en el contexto de la práctica de ejercicio físico. De igual forma, se hipotetizó que respecto a los chicos, las chicas mostrarían mayores niveles de riesgo de padecer un TCA (Ferreiro et al., 2012; Herpertz-Dahlmann et al., 2015; Veses et al., 2014).

\section{Método}

\section{Participantes}

Participaron 236 escolares (117 chicos y 119 chicas) de entre 13 y 17 años de edad $\left(M_{\text {edad }}=\right.$ $13.78, D T=1.15)$ pertenecientes a dos centros educativos de una provincia del sur de España. De éstos, 71 (30\%) cursaban primer curso de ESO, 65 (27\%) segundo curso, 80 (34\%) tercer curso, y 20 (9\%) cuarto curso.

\section{Instrumentos}

Comparación de la Apariencia al Realizar Ejercicio Físico. Se utilizó una adaptación al español del ítem número 11 (When I'm at the gym, I compare my physical appearance to the appearance of others) de la Physical Appearance Comparison Scale-Revised (PACS-R; Schaefer \& Thompson, 2014). Atendiendo al objetivo planteado, el término "gimnasio" fue sustituido por el más genérico "ejercicio físico" (i.e., Cuando estoy haciendo ejercicio físico comparo mi apariencia con la del resto de la gente). La respuesta se realizó utilizando una escala Likert que oscilaba de 0 (nunca) a 4 (siempre).

Insatisfacción Corporal. Se utilizó una adaptación al español (¿En qué grado te sientes insatisfecho/a con tu peso o tu figura?) del ítem (Over the past 7 days, how dissatisfied have you been with your weight or shape?) originalmente propuesto por Gideon et al. (2016). La respuesta se realizó utilizando una escala Likert que oscilaba de 0 (En absoluto) a 3 (Considerablemente).

Riesgo de Presencia de Trastornos de la Conducta Alimentaria. Se utilizó la versión española (Garcia-Campayo et al., 2005) del cuestionario de cribado SCOFF (Morgan, Reid, \& Lacey, 1999). El instrumento se compone de cinco ítems (e.g., ¿Dirías que la comida domina tu vida?) que se responden de forma dicotómica $(N o=0, S i=1)$. Mayores puntuaciones sugieren mayor riesgo de presencia de TCA. Utilizado como herramienta de cribado, puntuaciones iguales o superiores a dos sugieren la posible presencia de un TCA.

Cuestionario socio-demográfico. Se solicitó a los participantes que informasen del curso al que asistían, así como de su sexo, edad, estatura, y peso.

\section{Análisis de datos}

En primer lugar, se calcularon los estadísticos descriptivos (media, desviación típica, asimetría, y apuntamiento), así como las correlaciones bivariadas entre las variables de estudio. Seguidamente, se realizó una prueba $t$ de Student aplicando una técnica no paramétrica de bootstrapping de 10,000 remuestreos (Delaigle, Hall, \& Jin, 2011) para comprobar la posible existencia de diferencias en función del sexo de los participantes en: a) la frecuencia en la comparación de la apariencia al realizar ejercicio físico; y b) el riesgo de presencia de TCA. El tamaño del efecto de las diferencias observadas entre grupos $(d)$ se juzgó de acuerdo a los criterios y puntos de corte propuestos por Cohen (1988) (i.e., 0.2 pequeño, 0.5 moderado, 0.8 grande). Seguidamente, se realizó un análisis de regresión utilizando el macro PROCESS para el programa estadístico IBM SPSS v. 22 aplicando una técnica no paramétrica de bootstrapping de 10,000 remuestreos (Hayes, 2013). En dichos análisis, la frecuencia en la comparación de la apariencia al realizar ejercicio físico fue introducida como variable independiente y el riesgo de presencia de TCA como variable dependiente. A su vez, sexo, edad, IMC, e insatisfacción corporal fueron introducidas como covariables, siendo 
adicionalmente consideradas como posibles variables moderadoras en la relación entre la frecuencia en la comparación de la apariencia al realizar ejercicio físico y el riesgo de presencia de TCA (ver Figura 1). Se estableció un nivel de significación estadística de $p<.05$ para la totalidad de análisis realizados.

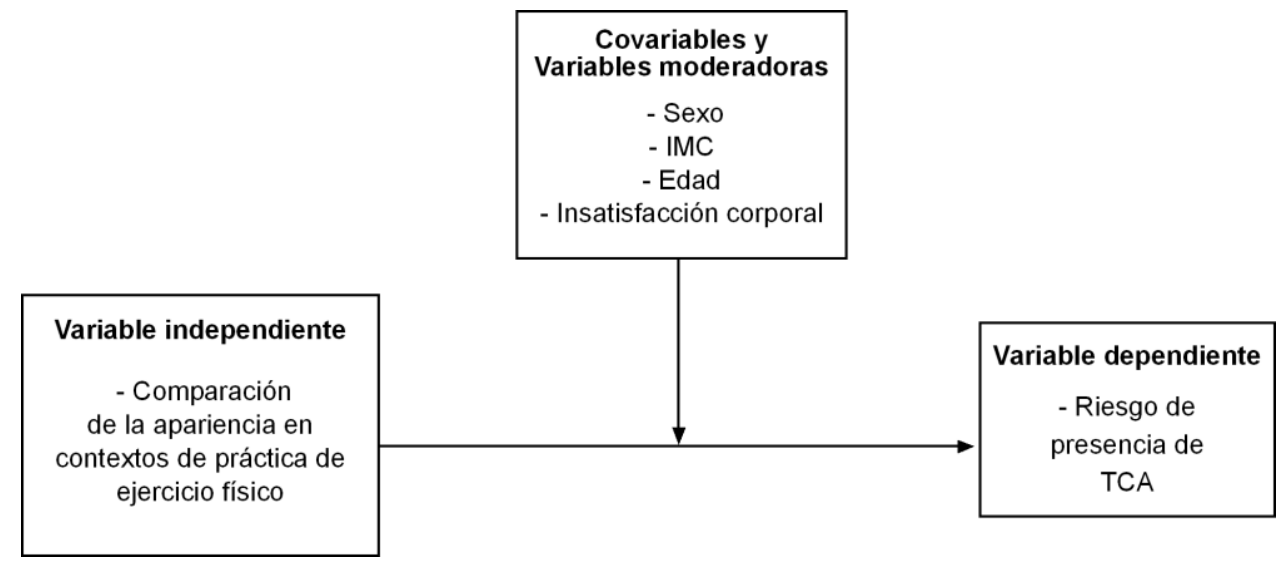

Figura 1. Modelo de moderación que considera el papel de sexo, IMC, edad, e insatisfacción corporal en la relación entre la comparación de la apariencia realizada en contextos de práctica de ejercicio y el riesgo de presencia de TCA.

\section{Resultados}

\section{Análisis preliminares}

La Tabla 1 muestra la existencia de correlaciones positivas y estadísticamente significativas entre la totalidad de variables consideradas y el riesgo de presencia de TCA. A su vez, las puntuaciones de insatisfacción corporal, comparación de la apariencia al realizar ejercicio físico y riesgo de presencia de TCA se situaron, en todo caso, por debajo del punto medio de la escala, sugiriendo por tanto la presencia de bajos niveles para dichas variables. Los resultados de las pruebas $t$ de Student mostraron puntuaciones más altas en chicas que en chicos para la comparación de la apariencia al realizar ejercicio físico $(t[234]=0.273$, IC95\% para la diferencia de medias $=-0.279$ a $0.382, p=.785, d=0.04)$, riesgo de presencia de TCA $(t[224.061]=1.003$, IC95\% para la diferencia de medias $=-0.141$ a $0.434, p=.317, d=0.14$ ); no obstante, dichas diferencias no fueron estadísticamente significativas en ninguno de los casos, siendo a su vez muy reducida la magnitud del tamaño del efecto. A su vez, un $29 \%$ de las chicas y un $21 \%$ de los chicos fueron clasificados como en riesgo de padecer un TCA de acuerdo a los puntos de corte sugeridos para el SCOFF (Morgan et al. 1999).

Tabla 1.

Estadísticos Descriptivos y Correlaciones Bivariadas entre Variables.

\begin{tabular}{|c|c|c|c|c|c|c|c|c|c|}
\hline & $\begin{array}{l}\text { Rango } \\
\text { posible }\end{array}$ & $M$ & $D T$ & Asimetría & Apuntamiento & 1 & 2 & 3 & 4 \\
\hline 1. Edad & - & 13.780 & 1.151 & 0.940 & -0.572 & & & & \\
\hline 2. IMC & - & 20.429 & 3.704 & 1.165 & 2.574 & $.135^{*}$ & & & \\
\hline 3. Insatisfacción corporal & $0-3$ & 1.100 & 1.008 & 0.557 & -0.774 & $-.151 *$ & $.288 * * *$ & & \\
\hline 4. CAEF & $0-4$ & 1.260 & 1.304 & 0.698 & -0.718 & -.037 & .059 & $.278 * * *$ & \\
\hline $\begin{array}{l}\text { 5. Riesgo de presencia de } \\
\text { TCA }\end{array}$ & $0-5$ & 0.877 & 1.121 & 1.268 & 1.129 & $.134 *$ & $.340 * * *$ & $.470 * * *$ & $.304 * * *$ \\
\hline
\end{tabular}

Nota IMC = Índice de masa corporal; $\mathrm{CAEF}=$ Comparación de la apariencia al realizar ejercicio físico; TCA = Trastorno de la conducta alimentaria. $* p<.05 ; * * p<.01 ; * * * p<.001$. 


\section{Análisis principales}

Los resultados de los análisis de regresión (Tabla 1) muestran que, con independencia de los efectos de variables como sexo, edad, IMC, e insatisfacción corporal, la frecuencia en la comparación de la apariencia al realizar ejercicio físico explicaba de forma estadísticamente significativa el riesgo de presencia de TCA $(B=.138$, IC95\% = .042 a $.234, p=.005)$. A su vez, los resultados de los análisis de moderación muestran que, de entre la totalidad de posibles variables consideradas, tan solo la interacción con la percepción de insatisfacción corporal contribuía de forma estadísticamente significativa al modelo $(F[1,229]=14.213, p<.001)$, y que ésta incrementaba la varianza explicada en el riesgo de presencia de un TCA en un $4 \%$. En conjunto, el modelo propuesto explicó un 35\% de la varianza del riesgo de presencia de TCA $(F[6,229]=20.394, p<.001)$. La Figura 2 muestra que la influencia positiva de la comparación de la apariencia al realizar ejercicio físico sobre el riesgo de presencia de TCA se veía incrementada de manera estadísticamente significativa en presencia de mayores niveles de insatisfacción corporal. En concreto, los análisis post-hoc realizados empleando la técnica de Johnson-Neyman muestran que la interacción era significativa para valores de insatisfacción corporal iguales o superiores a .88 (en una escala de 0 a 3), dentro de los cuales se situaban el $67 \%$ de los participantes. Los resultados de los restantes análisis de moderación realizados, incluida la posibilidad de que la interacción entre la comparación de la apariencia y la insatisfacción difiriese en función del sexo de los participantes, no mostraron la existencia de ninguna otra interacción estadísticamente significativa.

Tabla 2.

Análisis de Regresión que Predice el Riesgo de Presencia de TCA.

\begin{tabular}{lcrrrrrr}
\hline & & & & & \multicolumn{2}{c}{ IC95\% $B$} \\
\cline { 6 - 8 } & $B(\beta)$ & ET $B$ & $t$ & $p$ & Inferior & Superior \\
\hline Sexo & $-.155(-.134)^{\mathrm{a}}$ & .122 & -1.277 & .203 & -.395 & .084 \\
Edad & $.068(.070)$ & .054 & 1.276 & .203 & -.037 & .174 \\
IMC & $.068(.225)$ & .017 & 4.006 & $<.001$ & .035 & .101 \\
CAEF & $.138(.160)$ & .049 & 2.826 & .005 & .042 & .234 \\
Insatisfacción corporal & $.340(.305)$ & .066 & 5.146 & $<.001$ & .210 & .470 \\
Interacción CAEF - Insatisfacción corporal & $.165(.194)$ & .044 & 3.770 & $<.001$ & .079 & .252 \\
\hline
\end{tabular}

Nota . IMC = Índice de masa corporal CAEF = Comparación de la apariencia al realizar ejercicio físico; $B=$ coeficientes de regresión no estandarizados; $\beta=$ coeficientes de regresión estandarizados; ET = error típico; IC = Intervalo de confianza. Análisis realizado aplicando una técnica de bootstrapping de 10,000 remuestreos. ${ }^{a}$ Valores negativos indican la presencia de menores niveles en chicos respecto a chicas.

\section{Discusión}

El principal objetivo de este estudio fue observar la posible influencia de la comparación de la apariencia en el contexto de la práctica de ejercicio físico sobre el riesgo de presencia de TCA en población en adolescente, considerando a su vez el posible papel moderador que en dicha relación podrían jugar algunas variables previamente asociadas con este desorden. Los resultados obtenidos confirmaron la hipótesis principal planteada. Además, éstos se muestran relevantes por cuanto aportan por primera vez evidencias que apoyan la relación entre la frecuencia en la comparación de la apariencia durante la práctica de ejercicio físico y el riesgo de presencia de TCA en adolescentes de ambos sexos, a la vez que sugieren que dicha relación podría verse acentuada por la percepción de insatisfacción corporal.

Los resultados del presente trabajo se muestran en línea con los de anteriores estudios que informaron de la capacidad predictiva incremental que la frecuencia en la comparación social de la propia apariencia respecto al IMC considerando muestras integradas exclusivamente por mujeres en las últimas fases de la adolescencia y adultas (Fitzsimmons-Craft et al., 2012; Fitzsimmons-Craft et al. 
2014; Schaefer \& Thompson, 2014). De igual forma, los resultados obtenidos apoyan la relación positiva previamente observada entre la frecuencia en la comparación de la apariencia considerada de forma genérica y el riesgo de presencia de TCA en población adolescente de ambos sexos (Halliwell \& Harvey, 2006), en el caso del presente estudio, circunscrita dicha tendencia a la comparación al ámbito específico de la práctica de ejercicio físico.

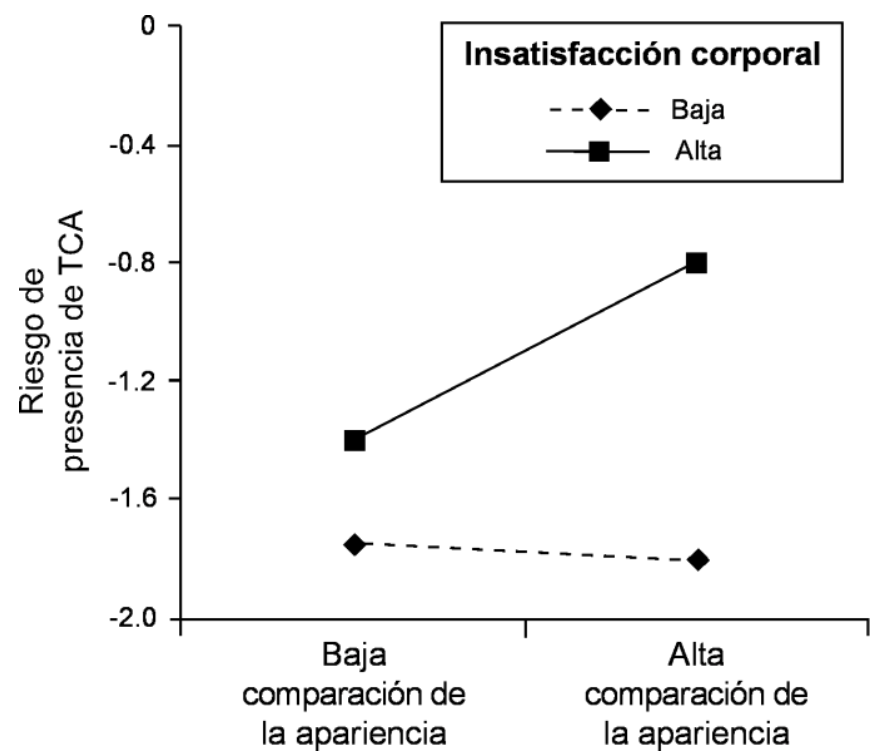

Figura 2. Interacción de dos-vías entre la frecuencia de comparación de la apariencia en contextos de práctica de ejercicio físico e insatisfacción corporal sobre los síntomas de presencia de TCA. Las pendientes de regresión fueron derivadas de ecuaciones de regresión considerando valores situados una desviación típica por encima (alto nivel) y por debajo (bajo nivel) de la media tanto para la variable independiente (comparación de la apariencia) como para la moderadora (insatisfacción corporal) (Hayes, 2013).

Al igual que en anteriores investigaciones realizadas en adolescentes de edad similar a la considerada en el presente estudio (Halliwell \& Harvey, 2006), las chicas mostraron niveles superiores de comparación de la apariencia respecto a los chicos, en este caso, en el contexto de la práctica de ejercicio físico. No obstante, el tamaño del efecto observado fue muy bajo, sugiriendo por tanto la práctica ausencia de diferencias en este sentido. Estos resultados sugieren que, al menos en el contexto de la práctica de ejercicio físico, la tendencia a comparar la propia apariencia podría producirse de forma muy similar en chicas que en chicos, hecho que vendría a plantear la necesidad de profundizar en el estudio de los antecedentes y posibles consecuencias derivadas de dicha tendencia en población adolescente de ambos sexos.

En línea con los resultados de anteriores investigaciones (Fitzsimmons-Craft et al., 2012; Fitzsimmons-Craft et al. 2014; Schaefer \& Thompson, 2014), las chicas presentan un mayor riesgo de padecer un TCA que los chicos de acuerdo al punto de corte considerado como herramienta de cribado del cuestionario SCOFF (29 vs 21\%). Sin embargo, las diferencias en función del sexo en las puntuaciones globales para el riesgo de desarrollar un TCA obtenidas en el presente estudio no fueron estadísticamente significativas. Estos resultados ponen de manifiesto la necesidad de profundizar en el estudio de los factores que conducen a la aparición de los TCA también en población masculina durante las primeras etapas de la adolescencia.

Desde una perspectiva práctica, los resultados obtenidos permiten identificar un elemento de riesgo en la aparición de posibles TCA. De esta forma, se aportan evidencias que sugieren que la frecuencia en la comparación de la apariencia al realizar ejercicio físico debería ser contemplada en el diseño de programas destinados a la prevención de este tipo de desórdenes; en especial, atendiendo al efecto de interacción observado con la insatisfacción corporal, elemento este último especialmente presente en población adolescente y previamente identificado de manera prospectiva como uno de los mejores predictores en el riesgo de aparición de TCA (Ferreiro et al., 2012; Rohde et al., 2015). A su 
vez, de las evidencias obtenidas podrían derivarse ciertas recomendaciones aplicables a la práctica profesional tanto de profesores de Educación Física como de monitores o entrenadores deportivos que trabajan con población adolescente. En concreto, los resultados del presente estudio ponen de relieve la necesidad de que dichos profesionales no favorezcan con su actuación la comparación de la apariencia en dicho contextos, por ejemplo, contemplando esta posibilidad a la hora de diseñar las diferentes programaciones didácticas. En esta misma línea, otra posible propuesta sería evitar, en la medida de lo posible, establecer referentes o modelos dentro del grupo respecto a quienes el alumnado deba compararse.

Pese a que los resultados obtenidos ofrecen una serie de evidencias que podrían resultar de utilidad en la prevención de la aparición de TCA en adolescentes, el presente estudio contó con algunas limitaciones que deben ser reseñadas. En primer lugar, cabe destacar que aunque la brevedad de los instrumentos utilizados para medir la frecuencia de la comparación de la apariencia o el grado de insatisfacción con el propio peso y forma corporal resultaba deseable considerando el carácter adolescente de la población en la cual fueron aplicados (Gogol et al., 2014), no existen evidencias previas que apoyen su uso frente a instrumentos específicos de mayor extensión. En segundo lugar, aunque el ítem utilizado para medir la insatisfacción corporal aludía a dos componentes de la insatisfacción corporal previamente identificados en la literatura (i.e., peso y figura; Neighbors \& Sobal, 2007), éste lo hacía de manera conjunta, hecho que impidió comprobar si, al igual que sugieren anteriores investigaciones (Guszkowska \& Mosur-Kałuża, 2015), ambos componentes contribuían a explicar de manera diferenciada y complementaria otras variables, en este caso, el riesgo de presencia de TCA. En tercer lugar, cabe destacar que el instrumento utilizado para medir la frecuencia en la comparación de la apariencia no permitía distinguir entre los distintos atributos que podrían ser sometidos a comparación. Esta limitación podría resultar relevante por cuanto anteriores estudios han puesto de manifiesto el carácter diferenciado de las consecuencias derivadas de la comparación de la apariencia considerada de forma general respecto a la comparación de elementos específicos como el peso o el nivel de desarrollo muscular (McCreary \& Saucier, 2009). Finalmente, debe reseñarse que el carácter transversal del presente estudio impidió establecer un orden causal entre las variables consideradas.

Al objeto de confirmar y ampliar el alcance de los resultados del presente trabajo, futuros estudios podrían considerar la posible influencia de elementos específicos de la comparación de la apariencia directamente relacionados con los modelos corporales asociados tradicionalmente en mayor medida a chicas (i.e., cuerpo delgado) y chicos (cuerpo musculoso) (Flament et al., 2012). Este aspecto podría resultar de interés, sobre todo, en función de las evidencias que sugieren que el ideal de cuerpo delgado podría estar presente en igual medida en adolescentes de ambos sexos y edad similar a los considerados en el presente estudio (Granero-Gallegos, Martín-Albo, Sicilia, Medina-Casaubón, \& Alcaráz-Ibáñez, 2017). Otra posible línea de investigación a abordar en futuros trabajos sería estudiar la influencia que sobre el riesgo de presencia de TCA podría ejercer la comparación de la apariencia considerando de manera diferenciada los distintos contextos de práctica de ejercicio físico presentes de manera implícita en el presente estudio (i.e., clases de educación física, práctica de ejercicio físico durante el tiempo libre o, incluso, la participación en actividades deportivas); de esta forma, se podrían obtener evidencias que permitiesen acotar en mayor medida el ámbito en el cual focalizar los esfuerzos destinados a paliar la tendencia a la comparación de la apariencia como una medida de prevención de la aparición de los TCA.

En conclusión, los resultados del presente estudio sugieren que la frecuencia de la comparación de la apariencia realizada en los contextos de práctica de ejercicio físico por parte de los adolescentes se muestra como un elemento que podría contribuir a explicar el riesgo de presencia de TCA, tanto en chicas como en chicos y, en especial, en aquellos adolescentes más insatisfechos con su peso y figura. No obstante, estos hallazgos deben ser considerados como unas primeras evidencias de una relación que debería ser objeto de estudio de futuros estudios. 


\section{Referencias}

Cohen, J. (1988). Statistical Power Analysis for the Behavioral Sciences (2nd ed.). Hillsdale, New Jersey: Lawrence Erlbaum.

Delaigle, A., Hall, P., \& Jin, J. (2011). Robustness and accuracy of methods for high dimensional data analysis based on Student's t-statistic. Journal of the Royal Statistical Society: Series B (Statistical Methodology), 73(3), 283-301.

DiClemente, R. J., Hansen, W. B., \& Ponton, L. (1996). Handbook of adolescent health risk behavior. New York: Plenum.

Ferreiro, F., Seoane, G., \& Senra, C. (2012). Gender-related risk and protective factors for depressive symptoms and disordered eating in adolescence: A 4-year longitudinal study. Journal of Youth and Adolescence, 41(5), 607-622. http://doi.org/10.1007/s10964-011-9718-7.

Festinger, L. (1954). A theory of social comparison processes. Human Relations, 7, 117-140.

Fitzsimmons-Craft, E. E., Bardone-Cone, A. M., Bulik, C. M., Wonderlich, S. A., Crosby, R. D., \& Engel, S. G. (2014). Examining an elaborated sociocultural model of disordered eating among college women: The roles of social comparison and body surveillance. Body Image, 11(4), 488-500. http://doi.org/10.1038/jid.2014.371.

Fitzsimmons-Craft, E. E., Bardone-Cone, A. M., \& Harney, M. B. (2012). Development and validation of the Body, Eating, and Exercise Comparison Orientation Measure (BEECOM) among college women. Body Image, 9(4), 476-487. http://doi.org/10.1016/j.bodyim.2012.07.007.

Flament, M. F., Hill, E. M., Buchholz, A., Henderson, K., Tasca, G. A., \& Goldfield, G. (2012). Internalization of the thin and muscular body ideal and disordered eating in adolescence: The mediation effects of body esteem. Body Image, 9(1), 68-75.

Garcia-Campayo, J., Sanz-Carrillo, C., Ibañez, J. A., Lou, S., Solano, V., \& Alda, M. (2005). Validation of the Spanish version of the SCOFF questionnaire for the screening of eating disorders in primary care. Journal of Psychosomatic Research, 59(2), 51-55. http://doi.org/10.1016/j.jpsychores.2004.06.005.

Gideon, N., Hawkes, N., Mond, J., Saunders, R., Tchanturia, K., \& Serpell, L. (2016). Development and psychometric validation of the EDE-QS, a 12 item short form of the Eating Disorder Examination questionnaire (EDE-Q). PLoS ONE, 11(5), 1-19. http://doi.org/10.1371/journal.pone.0152744.

Gogol, K., Brunner, M., Goetz, T., Martin, R., Ugen, S., Keller, U., ... Preckel, F. (2014). "My questionnaire is too long!" The assessments of motivational-affective constructs with three-item and single-item measures. Contemporary Educational Psychology, 205. http://doi.org/10.1016/j.cedpsych.2014.04.002.

Granero-Gallegos, A., Martín-Albo, J., Sicilia, A., Medina-Casaubón, J., \& Alcaráz-Ibáñez, M. (2017). Analysis of sociocultural stereotypes towards thin body and muscular body: Differences according to sex and weight discrepancy. Journal of Psychodidactics, 22(2), 1-14. http://doi.org/10.1387/RevPsicodidact.17182.

Guszkowska, M., \& Mosur-Kałuża, S. (2015). Determinants of women's willingness to continue fitness training in spite of health risks. Biomedical Human Kinetics, 7(1), 51-57. http://doi.org/10.1515/bhk-2015-0009.

Halliwell, E., \& Harvey, M. (2006). Examination of a sociocultural model of disordered eating among male and female adolescents. British Journal of Health, 11, 235-248. http://doi.org/10.1348/135910705X39214.

Hayes, A. F. (2013). Introduction to mediation, moderation, and conditional process analysis: A regressionbased approach. New York, NY: Guilford Press.

Herpertz-Dahlmann, B., Dempfle, A., Konrad, K., Klasen, F., \& Ravens-Sieberer, U. (2015). Eating disorder symptoms do not just disappear: The implications of adolescent eating-disordered behaviour for body weight and mental health in young adulthood. European Child and Adolescent Psychiatry, 24(6), 675684. http://doi.org/10.1007/s00787-014-0610-3.

Klump, K. L. (2013). Puberty as a critical risk period for eating disorders: A review of human and animal studies. Hormones and Behavior, 64(2), 399-410. http://doi.org/10.1016/j.yhbeh.2013.02.019.

Mayer-Brown, S., Lawless, C., Fedele, D., Dumont-Driscoll, M., \& Janicke, D. M. (2016). The effects of media, self-esteem, and BMI on youth's unhealthy weight control behaviors. Eating Behaviors, 21, 59-65. http://doi.org/10.1016/j.eatbeh.2015.11.010.

McCreary, D. R., \& Saucier, D. M. (2009). Drive for muscularity, body comparison, and social physique anxiety in men and women. Body Image, 6(1), 24-30. http://doi.org/10.1016/j.bodyim.2008.09.002. 
Morgan, J. F., Reid, F., \& Lacey, J. H. (1999). The SCOFF questionnaire: Assessment of a new screening tool for eating disorders. $B M J, 319,1467-1468$. http://doi.org/10.1136/bmj.319.7223.1467.

Neighbors, L. A., \& Sobal, J. (2007). Prevalence and magnitude of body weight and shape dissatisfaction among university students. Eating Behaviors, 8(4), 429-439. http://doi.org/10.1016/j.eatbeh.2007.03.003.

Rohde, P., Stice, E., \& Marti, C. N. (2015). Development and predictive effects of eating disorder risk factors during adolescence: Implications for prevention efforts. International Journal of Eating Disorders, 48(2), 187-198. http://doi.org/10.1002/eat.22270.

Schaefer, L. M., \& Thompson, J. K. (2014). The development and validation of the Physical Appearance Comparison Scale-Revised (PACS-R). Eating Behaviors, 15(2), 209-217. http://doi.org/10.1016/j.eatbeh.2014.01.001.

Veses, A. M., Gómez-Martínez, S., Pérez de Heredia, F., Esteban-Cornejo, I., Castillo, R., Estecha, S., ... Marcos, A. (2014). Cognition and the risk of eating disorders in Spanish adolescents: The AVENA and AFINOS studies. European Journal of Pediatrics, 174(2), 229-236. http://doi.org/10.1007/s00431-0142386-3.

Wang, M. L., Peterson, K. E., McCormick, M. C., \& Austin, S. B. (2013). Environmental factors associated with disordered weight-control behaviours among youth: A systematic review. Public Health Nutrition, 17(7), 1-14. http://doi.org/10.1017/S1368980013001407.

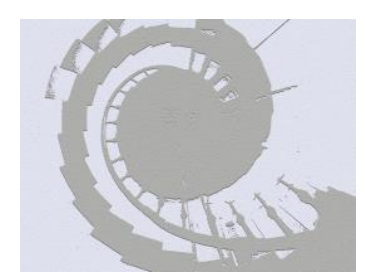

